This is a self-archived - parallel published version of this article in the publication archive of the University of Vaasa. It might differ from the original.

\title{
Reform der öffentlichen Dienste im internationalen Vergleich
}

Author(s): Demmke, Christoph

Title: $\quad$ Reform der öffentlichen Dienste im internationalen Vergleich

Year: $\quad 2019$

Version: Accepted manuscript

Copyright (C)2019 Springer Fachmedien Wiesbaden GmbH, ein Teil von Springer Nature. This is a post-peer-review, pre-copyedit version of an article published in Veit S., Reichard C., \& Wewer G. (eds), Handbuch zur Verwaltungsreform. The final authenticated version is available online at: https://doi.org/10.1007/978-3-658-21563-7_33

\section{Please cite the original version:}

Demmke, C., (2019). Reform der öffentlichen Dienste im internationalen Vergleich. In: Veit S., Reichard C., \& Wewer G. (eds), Handbuch zur Verwaltungsreform (pp. 373-383). Springer VS, Wiesbaden. https://doi.org/10.1007/978-3-65821563-7_33 


\title{
Reform der öffentlichen Dienste im internationalen Vergleich
}

\author{
Christoph Demmke
}

Keywords: Öffentlicher Dienst, Laufbahnsystem, Positionssystem, Personalmanagement; Europäischer Verwaltungsraum

\begin{abstract}
Die öffentlichen Dienste in Europa haben sich im Laufe der Zeit von einem stärker am Bürokratiemodell angelehnten Konzept $\mathrm{zu}$ sehr unterschiedlichen Personalsystemen entwickelt. Der Beitrag stellt Begriffselemente des öffentlichen Dienstes vor, diskutiert die Unterschiede eines Laufbahn- und eines Positionssystems und präsentiert praktische Reformansätze sowie Umsetzungserfahrungen. Insgesamt wird deutlich, dass man in Europa mittlerweile kaum noch von einem universellen Modell des öffentlichen Dienstes sprechen kann.
\end{abstract}

\section{Einleitung}

In der Öffentlichkeit ist noch immer sehr wenig über die verschiedenen nationalen öffentlichen Dienste bekannt. Analytische Vergleichsstudien sind selten (aber Auer et al. 1996; Verheijen 1999; Bossaert et al. 2001; Demmke and Moilanen 2010, 2013; van der Meer et al. 2011, 2015; Meyer-Sahling 2012; Meyer-Sahling et al. 2016), obwohl sich ein Vergleich seit dem Auftauchen von bürokratischen Verwaltungssystemen in nahezu allen Staaten geradezu anbietet. Die damals entstandenen öffentlichen Dienste symbolisierten einen Rationalisierungsschub der Moderne und der Aufklärung und zugleich eine Abgrenzung im Verhältnis zur politischen Macht. In allen Staaten Europas wurden die öffentlichen Dienste als maschinenähnliche, hierarchische, de-personalisierte und standardisierte Systeme konzipiert, die keinem partikularen Interesse, sondern vor allem dem Recht und der staatlichen Aufgabenerfüllung dienen sollten. In den letzten Jahren kommt es allerdings zu einer immer stärkeren Ausdifferenzierung von Organisationsstrukturen und Beschäftigungsmodellen, immer neuen Modellen und Verfahren staatlicher und nicht-staatlicher Aufgabenerfüllung sowie Kritik am Rationalitätsparadigma schlechthin. In der Folge kann von der Existenz eines europaweiten Konzepts des öffentlichen Dienstes keine Rede mehr sein. 


\section{Definition der öffentlichen Dienste}

Ein Vergleich der europäischen öffentlichen Dienste stößt zunächst auf linguistische Schwierigkeiten. Die Begriffe „öffentlicher Dienst“, „öffentliche Verwaltung“ und „Beamtentum“ werden international und europaweit sehr uneinheitlich gehandhabt. Bisher gibt es weder eine internationale, noch eine EU-weit anerkannte Definition des öffentlichen Dienstes. Somit ist es ebenfalls schwierig, eine europaweite Definition des Begriffes „Beamter“ vorzunehmen, obwohl die Rechtsprechung des Europäischen Gerichtshofes dazu beigetragen hat, den Begriff „Arbeitnehmer“ mit Blick auf Art. 45 Abs. 4 EUV zu definieren.

Um nur einige Beispiele zu nennen: der Begriff des Beamtentums wird durch „Civil Service“ in Großbritannien, ,fonction publique“ in Frankreich und „Overheidsdienst“ oder „Ambtelijk Apparat " in den Niederlanden wiedergegeben. Alle Begriffe sind jedoch nicht deckungsgleich. So betrifft die britische Definition des „Civil Servant“ einen Bediensteten der Krone in ziviler Stellung, der kein politisches oder richterliches Amt innehat. Dabei handelt es sich aber nur um einen kleinen Teil der öffentlichen Bediensteten. Auch in Irland und in Polen betrifft der Begriff des „Civil Servants“ nur einen sehr eingeschränkten Kernbereich der öffentlichen Beschäftigten, in der Regel auf zentraler Staatsebene. Dagegen gibt es in Frankreich drei „Fonctions Publiques“. Im Gegensatz zum Vereinigten Königreich mit ca. 450.000 Crown Civil Servants können in Frankeich etwa fünf Millionen öffentliche Bedienstete als Beamte (fonctionnaires titulaires) bezeichnet werden, also etwa zehnmal so viele wie in Großbritannien. In den Niederlanden umfasst der Begriff „Overheidsdienst“ das gesamte öffentliche Personal einschließlich der weitgehend unabhängigen Wasseragenturen und kann am ehesten als Öffentlicher Dienst übersetzt werden. Aufgrund des (weitestgehend) einheitlichen Dienstrechts sind in den Niederlanden - wie in Frankreich - fast alle Beschäftigten im öffentlichen Dienst zugleich Beamte. Dabei unterscheiden sich die Beschäftigungsverhältnisse der niederländischen Beamten kaum von den Beschäftigten im Privatsektor. Hier wird der enorme Unterschied zwischen dem niederländischen und bspw. dem französischen Konzept deutlich, da sich in Frankreich, anders als in den Niederlanden, die Beschäftigungsverhältnisse zwischen Beamten und Angestellten (wie in Deutschland) grundsätzlich unterscheiden. Zudem erfordert in Frankreich die Rekrutierung ein spezielles Zugangsverfahren in einem so genannten Concours. Diese kurzen Länderbeispiele können die enormen Unterschiede nur andeuten, die sich bei einer europäischen Übersicht in den 28 Mitgliedstaaten der EU erkennen lassen. Öffentliche Dienste, bei denen die gesamte öffentliche Beschäftigung mit der Beamtenbeschäftigung weitgehend deckungsgleich ist, gibt es in Kroatien (93\%), den Niederlanden (90\%), Griechenland (90\%), und mit Abstrichen in Finnland, Irland und Belgien 
auf der zentralen Staatsebene. In allen anderen Mitgliedstaaten variiert der Anteil der Beamten an der öffentlichen Beschäftigung zwischen $0,5 \%$ (Schweden), $5 \%$ (Polen), $37 \%$ (Deutschland), 70 \% (Zypern) sowie $75 \%$ in Frankreich. In der Schweiz sind auf Bundesebene nur noch Polizeibeamte, Richter und einige Finanzbedienstete Beschäftigte mit Beamtenstatus. In den meisten Mitgliedstaaten konzentriert sich die Beamtenbeschäftigung auf die Kernbereiche hoheitlichen Handelns und pendelt sich bei $20 \%$ bis $50 \%$ der öffentlichen Beschäftigung ein (European Commission 2018).

Ein Vergleich der öffentlichen Dienste zeigt zudem enorme Unterschiede bei Status und Beschäftigungsbedingungen. Während sich in einzelnen Staaten die Beschäftigungsbedingungen in den öffentlichen Diensten kaum noch von denen im Privatsektor unterscheiden, halten andere Staaten, wie z. B. Deutschland und Frankreich, an den klassischen Grundsätzen des bürokratischen Berufsbeamtentums fest, mit zum Teil noch immer großen Unterschieden zwischen Beamten und Angestellten. Diese Unterschiede sind schon auf kleinstem geographischem Raum (und auch zunehmend im innerstaatlichen Bereich) feststellbar. So hat die Schweiz den Beamtenstatus weitestgehend abgeschafft, in Österreich wird von der „Pragmatisierung“ (Verbeamtung) nur noch eingeschränkt Gebrauch gemacht, während in Deutschland das Beamtentum erst kürzlich durch die Rechtsprechung des BVerfG (2 BvR 1738/12, 2018) zum Streikverbot für Beamte eine erneute Legitimierung erfahren hat. Auch auf praktischer Ebene gibt es große Unterschiede bei der Entscheidung, durch wen öffentliche Aufgaben erbracht werden sollen. Diese Unterschiede sind an den Beschäftigtenzahlen im öffentlichen Dienst in Europa ablesbar, die, gemessen an der Gesamtbeschäftigung, nunmehr zwischen ca. $13 \%$ und $30 \%$ ausmachen. Im Übrigen variieren die Zahlen, je nachdem welche Definition des öffentlichen Dienstes zugrunde gelegt wird. Man denke etwa an eine sehr breite Konzeption des öffentlichen Dienstes, die die öffentlichen Unternehmen integriert, an ein Konzept mit mittlerer Reichweite, welches öffentliche Unternehmen ausschließt sowie an eine enge Konzeptualisierung, bei der der Gesundheits-, Bildungs-, Militär-, Polizei- und Sozialversicherungsbereich herausgerechnet wird.

\section{Charakteristika der Öffentlichen Dienste in Europa: Laufbahn- und Positionssysteme}

Internationale Vergleichsstudien kategorisieren die unterschiedlichen Personalsysteme häufig in Laufbahnsysteme und Positionssysteme.

\subsection{Laufbahnsystem}


Laufbahnsysteme sind Beamtensysteme, die sich von den privatrechtlichen Arbeitsbedingungen abheben. In den vergleichenden Verwaltungswissenschaften werden viele klassische öffentliche Dienste noch immer als Laufbahnsysteme eingestuft (Kuhlmann und Wollmann 2014; European Commission 2018, p. 24). Solche Systeme werden dadurch charakterisiert, dass Beamte, die nach Abschluss ihrer Ausbildung in den öffentlichen Dienst eintreten, dort ihr Berufsleben bis zum Erreichen des Eintrittsalters in den Ruhestand verbringen werden. Eine Kündigung ist nur aufgrund disziplinarrechtlicher Gründe möglich, zudem ist das Streikrecht nicht vorgesehen. Der öffentliche Dienst ist in einer Weise strukturiert, die dem Beamten die Verfolgung einer Berufslaufbahn innerhalb des öffentlichen Dienstes ermöglicht. So erfolgt die Rekrutierung in einem Eingangsamt und Beförderungen geschehen in einem streng geordneten hierarchischen Verfahren. Daher gibt es in allen Laufbahnsystemen spezielle Rekrutierungsverfahren (d. h. die erfolgreiche Ableistung eines Concours, ein Staatsexamen mit oder ohne Vorbereitungsdienst, spezielle Fortbildungserfordernisse etc.). Die Einstellungsverfahren verlaufen sehr formalisiert, um einen hohen Grad an Objektivität und Gleichbehandlung zu erzielen. Weitere charakteristische Merkmale dieses Systems sind die Verleihung eines Dienstgrades an den Beamten sowie die Trennung von Dienstgrad und Stelle. Der Beamte wird nach dem Erfahrungs- bzw. Dienstaltersprinzip befördert und entlohnt und kann im Zuge seiner Laufbahn in der Hierarchie der Dienstgrade und Funktionsbezeichnungen aufsteigen. Berufserfahrung im privaten Sektor findet - aufgrund der Besonderheit einer Beschäftigung im öffentlichen Dienst - in der Regel nur eine beschränkte Anerkennung. Klassische Laufbahnkonzepte verfügen zudem über spezifische Sondersysteme der Altersversorgung und zuweilen auch der Krankenversorgung. Beim Laufbahnsystem unterscheiden sich der Status und die Beschäftigungsbedingungen der Beamten von den Beschäftigten im Privatsektor. Der öffentliche und der private Sektor sind dementsprechend vergleichsweise stark voneinander getrennt. In einem Laufbahnsystem soll die Arbeitsplatzgarantie (und in Deutschland das Alimentationsprinzip) die Kontinuität der vom Staat erbrachten Dienstleistungen sichern und vor politischer Einflussnahme (,spoil system“) schützen. Bedienstete in einem Laufbahnsystem werden daher auf Lebenszeit ernannt und können daher nur bei Vorliegen besonderer disziplinarrechtlicher Gründe entlassen werden.

\section{$\underline{3.2 \text { Positionssystem }}$}

In einer steigenden Anzahl an EU-Staaten gibt es diese Strukturelemente eines Laufbahnsystems nicht mehr. So können sich Bewerber aus dem privaten Bereich auf alle öffentlichen Stellen und Funktionen in einem offenen Wettbewerb bewerben. Rekrutierungen 
sowie Beförderungen sind zudem immer häufiger an kein Eingangs- oder Beförderungsamt gebunden. In einer Vielzahl von Mitgliedstaaten gibt es für den Bewerber somit auch kein stabiles Beförderungsmuster. Der Bedienstete muss die Stelle wechseln und sich auf eine neue Stelle bewerben, wenn er eine Entwicklung in seiner Karriere vollziehen möchte.

Insbesondere die skandinavischen Länder, die Schweiz, die Niederlande und Großbritannien lassen sich als öffentliche Dienste ohne Laufbahnstrukturen charakterisieren. Auch auf dem Gebiet der Altersversorgung und Krankenversicherung geht mittlerweile eine Mehrheit aller EU-Staaten von der Gleichheit der privaten und öffentlichen Beschäftigten aus. Der Mobilität zwischen dem Privatsektor und dem öffentlichen Dienst sind in immer mehr Mitgliedsstaaten der EU - zumindest theoretisch - keine Grenzen gesetzt. Folglich wird die Beschäftigung im öffentlichen Dienst als ein ganz normales Angestelltenverhältnis gesehen, das sich von einem privaten Dienstverhältnis nur noch wenig unterscheidet. Die besseren Möglichkeiten zur Mobilität zwischen dem öffentlichen und privaten Sektor erklären auch, warum eine vorausgehende Berufserfahrung im privaten Sektor in diesen Systemen in der Regel (auch was die Anrechnung der Pensionsrechte angeht) anerkannt wird. Mit Positionssystem ist somit grundsätzlich ein Personalsystem gemeint, in dem Beschäftigte jeweils für eine konkrete, spezifische Position bzw. Stelle eingestellt und eben nicht für eine gesamte Laufbahn rekrutiert werden. In den entsprechenden Staaten vertritt man die Auffassung, dass es keiner beamtenrechtlichen Sonderstrukturen bedarf, da die Politisierung des Personals sowie die Korruptionsanfälligkeit keinesfalls höher sei als in den klassischen Beamtenstaaten. Zwar wird die Auffassung akzeptiert, wonach die öffentlichen Dienste von essentieller Bedeutung sind und die Beschäftigten vor Politisierung, Korruption und Interessenkonflikten geschützt werden müssen. In der Regel geschieht dies vor allem durch arbeitsrechtliche Maßnahmen und Regelungen, aber auch durch die Schutzwirkungen von erhöhter Arbeitsplatzsicherheit, nicht aber durch das Laufbahn- und Lebenszeitprinzip, generelle Streikverbote, spezielle Pensionssysteme oder getrennte Krankenversicherungssysteme.

In vielen öffentlichen Diensten wurde in den letzten Jahren auch die Arbeitsplatzsicherheit relativiert. Während in Deutschland Beamte (grundsätzlich) nur aufgrund disziplinarrechtlicher Gründe entlassen werden können, gibt es in fast allen anderen Mitgliedstaaten zusätzliche Möglichkeiten, Beamte zu entlassen - etwa aufgrund der Haushaltslage, aufgrund von Restrukturierungsmaßnahmen, von spezifischen Organisationserfordernissen, wiederholten Schlechtleistungen bei Personalbeurteilungen etc. Damit ist das deutsche Modell auch hier in einer Sonderlage, zumindest was die Beamten angeht. Angesichts der steigenden Vielzahl an 
Beschäftigungsverhältnissen und befristeten Verträgen gilt allerdings auch in Deutschland der Öffentliche Dienst schon lange nicht mehr als Garant für Arbeitsplatzsicherheit.

Der europäische Status Quo lässt sich zusammenfassend wie folgt beschreiben: Die Unterschiede zwischen den öffentlichen Dienstmodellen sind zuweilen so groß, dass aus der Sicht eines deutschen Beamten z. B. die Situation der öffentlichen Beschäftigten in Schweden fast unvorstellbar sein dürfte: „Beamte“ können jederzeit auf allen Hierarchieebenen rekrutiert werden, es gibt ein weitgehendes Streikrecht, die Arbeitsverträge sind kündbar, die Besoldung wird individuell ausgehandelt, die Sozialpartner haben formelle Verhandlungsrechte und die Mobilität zwischen dem privaten und dem öffentlichen Dienst ist unbegrenzt. Insbesondere in Staaten mit Positionssystemen wird argumentiert, dass es auch im Tarif- und Arbeitsrecht genügend Instrumente gibt, mit denen der Sorge begegnet werden könnte, dass dann Mitarbeiter den Vorgesetzten oder gar der politischen Willkür ausgesetzt wären. Daneben gibt es ein weit reichendes Antidiskriminierungsrecht, Gleichbehandlungsvorschriften und Instrumente der kollektiven Rechtskontrolle. Insbesondere aus der Sicht der skandinavischen öffentlichen Dienste ist das Festhalten an Sonderstrukturen im öffentlichen Dienst fast gänzlich unnötig, da es keines Sondersystems bedarf, um Korruption und Politisierung zu reduzieren.

\section{Verwaltungstypologien und der Realitätsabgleich}

Ursprünglich wurden die diskutierten Einteilungen in Laufbahn- und Positionsmodelle vorgenommen (Auer et al. 1996), um die europäischen öffentlichen Dienste in Zeiten starker Veränderungen und immer neuen Reformmoden vergleichbar zu machen. Allerdings sind diese Typologien stark vereinfachend. Vielmehr ist gerade Deutschland ein Beispiel dafür, dass es immer schwieriger wird, nationale öffentliche Dienste zu vergleichen, weil die herkömmlichen Typen viel zu grob und zu wenig dynamisch erscheinen. Dies betrifft insbesondere die vorgenommene Unterscheidung zwischen Laufbahn- und Positionssystem. In der Realität können heute nur noch das luxemburgische, zypriotische, französische und deutsche System als Laufbahnsysteme gekennzeichnet werden und nehmen damit eine europäische Sonderstellung ein. Allerdings weisen selbst diese Systeme nicht alle klassischen Kriterien eines Laufbahnmodells auf. Insgesamt ist die Einteilung in Laufbahn- und Positionsmodelle zunehmend anachronistisch und viel $\mathrm{zu}$ allgemein, weil wichtige andere Attribute von Organisationsstrukturen und Arbeitssystemen nicht beachtet werden (z. B. Arbeitsbedingungen, Teamarbeit, Job Autonomie, Kompetenzerwerb, Führungsstil, kulturelle Faktoren und partizipative Formen der Arbeit). Zudem ist der Begriff des Positionssystems sehr vage, weil er eine Vielzahl völlig unterschiedlicher nationaler Systeme unter eine sehr 
allgemeine Begrifflichkeit subsumiert und zwar nur aufgrund der Tatsache, dass diese Systeme nicht mehr dem klassischen Beamtentypus entsprechen.

Grundsätzlich lassen sich nur schwer Aussagen über die Beziehungen zwischen institutioneller Struktur und Leistungsfähigkeit eines Systems des öffentlichen Dienstes machen. Zwar könnte man zunächst denken, dass sich ein weniger bürokratisiertes System des öffentlichen Dienstes positiv auf die Leistungsfähigkeit desselben auswirkt. Allerdings zeigt der Fall Deutschland, der in vielerlei Hinsicht ein vergleichsweise altmodisches Konzept des öffentlichen Dienstes aufweist, dass dieser Zusammenhang nicht unbedingt besteht. Mit Ausnahme des Bereiches der Digitalisierung gibt es keinen ernst zu nehmenden internationalen Verwaltungsvergleich, der Deutschland schlechte Noten verleiht, im Gegenteil. Insgesamt zeigt gerade das deutsche Beispiel, dass Aussagen über die Leistungsfähigkeit eines öffentlichen Dienstes nicht gemacht werden können, nur weil er vermeintlich altmodische Strukturelemente aufweist, oder nicht sogleich auf den neuesten Zug der Reformmoden aufgesprungen ist.

\section{Systemangleichungen in den öffentlichen Diensten}

In der Praxis sind das Laufbahn- und das Positionsmodell oft weniger weit voneinander entfernt als es zunächst scheint. Umgekehrt unterscheiden sich Staaten mit einem klassischen Laufbahnmodell untereinander mindestens so stark wie Staaten mit Positionsmodellen. Die Unterscheidung zwischen öffentlichem Dienstrecht und Arbeitsrecht ist häufig rein formalrechtlich. Aus materiell-rechtlicher Sicht können korrespondierende Beschäftigungsbedingungen sowohl durch Bestimmungen des öffentlichen Dienstrechts wie auch des Arbeitsrechts festgelegt werden. Gemeinsame Merkmale gibt es bspw. bei den Regelungen zur Arbeitszeit, im Krankheitsfall, Invalidität, Leistungsbeurteilung, bei der Anwendung des Gleichheits- und Leistungsprinzips, Anti-Diskriminierung, Bekämpfung der Korruption, Diversität und Vermeidung von Interessenkonflikten.

Auch in Staaten mit Positionssystemen treten Karrieremuster in Erscheinung, die den bürokratischen Laufbahnen durchaus ähnlich sind. Allerdings ist in einem Positionssystem die Beförderung zunächst ein Problem des Individuums, weil sich die Beschäftigten immer nur auf eine bestimmte Stelle bewerben können und es keine vorgegebenen Laufbahnentwicklungen gibt. Dagegen ist die Beförderung beim Laufbahnsystem ein Problem der Organisation, insbesondere wenn Beförderungsstellen fehlen oder Beschäftigte sich nicht auf Stellen bewerben können, die nicht in die Laufbahnlogik passen. 


\section{Ausgewählte Reform- und Umsetzungserfahrungen}

Unterschiedliche Reformprioritäten spiegeln zunächst einmal die (historische und institutionelle) Unterschiedlichkeit der öffentlichen Dienste sowie unterschiedliche Einflussfaktoren für Reformen wider. Beispielsweise spielt die Bewältigung des demographischen Wandels eine besonders wichtige Rolle in den Staaten mit den am stärksten alternden öffentlichen Diensten (Italien und Spanien). Geschlechterpolitiken sind weniger relevant in Staaten wie Polen, Lettland und Griechenland, weil in diesen Staaten ebenso viele Frauen wie Männer in Führungspositionen beschäftigt sind. Umgekehrt ist dieser Politikbereich in Belgien und den Niederlanden besonders wichtig, weil in diesen Staaten die Anzahl der Frauen in Spitzenpositionen nicht nur gering, sondern sogar rückläufig (OECD 2017, p. 97) ist. Vor allem während der Finanzkrise waren Reformstrategien nur in seltenen Fällen die Folge von rationalen Aufgabenkritiken und intelligenten Reformüberlegungen, sondern eher eine Folge von radikalen Austeritätspolitiken mit negativen Auswirkungen auf die Beschäftigten (Demmke 2016). So gibt es einen deutlichen Zusammenhang zwischen der Haushaltslage eines Staates und der (hohen) Anzahl an durchgeführten Sparmaßnahmen in den öffentlichen Diensten und dem Vertrauen der Beschäftigten in die Führung und Vorgesetzten (Demmke 2016; OECD 2016). Gerade die ökonomisch vergleichsweise erfolgreichen Staaten wie Norwegen und Deutschland zeichneten sich in den letzten Jahren eher durch Reformträgheit aus.

Ein eindeutiger Reformschwerpunkt betraf in fast allen öffentlichen Diensten das Ziel der Kosteneinsparungen. Diese Maßnahmen betrafen zum Beispiel das Einfrieren von Gehältern, das Nicht-Ersetzen von Beschäftigten, die in den Ruhestand wechselten sowie allgemeine Rekrutierungsstops. Trotz populärer Rhetorik, wonach Bildung, lebenslanges Lernen und Kompetenzentwicklung zentrale Elemente einer zukünftigen Personalpolitik sein sollen, wurde gerade in diesen Bereichen gespart. Im Bezahlungsbereich kam es in den letzten Jahren zu einer Aufspreizung und wachsenden Ungleichheit bei den Gehältern zwischen den einzelnen Beschäftigtenkategorien. Insgesamt ist der Abstand bei den Gehältern zwischen TopManagement und einfachem technischen Personal weiter gestiegen. Allgemein wird dies dadurch erklärt, dass Führung zunehmend stärker wertgeschätzt wird als technische Expertise. Die größten Gehaltsunterschiede gibt es in Italien und die geringsten in den Niederlanden. Insbesondere in Italien verspricht man sich durch die hohen Gehälter für Top-Beamte eine verbesserte Wettbewerbssituation mit dem Privatsektor und eine wirksame Maßnahme, um die Attraktivität von Managerpositionen im öffentlichen Dienst zu steigern. Hingegen wird die Wirkung dieser Maßnahmen in jenen Ländern eher kritisch gesehen. Zudem ist in einigen 
Staaten (z. B. in den Niederlanden) eine zu starke Gehälterspreizung im öffentlichen Dienst gesellschaftlich weniger vermittelbar als in stärker individualistischen Kulturen (vgl. zur Thematik der Wahrnehmung von Lohndisparität Brunner 2009).

In den letzten Jahren haben alle EU-Staaten den Grundsatz der automatischen Gehaltsanpassung und Beförderung nach Dienstalter, d. h. das Senioritätsprinzip, abgeschafft. Ebenfalls weitestgehend abgeschafft wurde die ausschließliche Rekrutierung von Bewerbern im Eingangsamt. Stattdessen spielt das Erfahrungsprinzip und das Kompetenzmanagement eine immer größere Rolle, obwohl bei letzterem nicht immer klar ist, wie individuelle Kompetenzen gemessen und bewertet werden sollen, wenn Qualifikationen und Noten als Bewertungsmaßstab an Bedeutung verlieren. Darüber hinaus wurde in vielen Staaten die Bezahlung und die Arbeitszeit flexibilisiert, die Arbeitsplatzsicherheit relativiert und die Personalzusammensetzung diversifiziert. Ein allgemeines Streikverbot für Beamte gibt es nur noch in sehr wenigen Staaten, darunter in Deutschland, nicht aber in der Schweiz. Im Bereich der personalbezogenen Organisationsreformen wurden in den letzten Jahren Zuständigkeiten vor allem im Bereich der leistungsorientierten Bezahlung, Fortbildung, Mobilität und Beförderung dezentralisiert (stark zentralisiert blieben nur die Zuständigkeiten im Bereich der Beschäftigungssteuerung und des Antidiskriminierungsrechts, im letzten Fall auch als Folge des Einflusses des Europarechts).

Bei der Individualisierung wird von den Beschäftigten im Bereich des lebenslangen Lernens und der persönlichen Kompetenzentwicklung gefordert, selbst mehr Verantwortung zu übernehmen. Gegenwärtige Kompetenzprofile beinhalten immer häufiger Anforderungen an Problemlösung, Selbstentwicklung, Lernen, Initiative, Innovation und Selbstverantwortung (OECD 2017b). Bei der Erbringung von individueller Leistung wird verstärkt auf intrinsische Motivation Wert gelegt und von den Beschäftigten mehr Engagement gefordert, insbesondere im Rahmen der so genannten Engagement Politics (OECD 2016).

\section{Trends und Herausforderungen}

Europaweit lässt sich kein Trend zu einem einheitlichen Modell des öffentlichen Dienstes feststellen. Im Gegenteil: die gegenwärtige Entwicklung erscheint zunehmend als Trend in Richtung eines „Flickenteppichdienstrechts“ mit unterschiedlichen Strukturen sowie Statusund Beschäftigungsbedingungen, teilweise auch innerstaatlich. In diesem Bereich stellen sich die größten Forschungsdefizite. So gibt es kaum vergleichende Untersuchungen über Arbeitssysteme und Arbeitsqualität, Arbeitsautonomie, Teamarbeit und Jobkontrolle, Lernförderung sowie vergleichende Analysen $\mathrm{zu}$ Kompetenzmessungen und 
Kompetenzveränderungen im öffentlichen Dienst. Dies gilt in besonderem Maße auch für den deutschen öffentlichen Dienst nach der Föderalismusreform.

Bei den Beamten halten viele Staaten nur noch an wenigen Sonderbedingungen fest. Diese konzentrieren sich auf die Bezahlung, Rekrutierung, erhöhte Arbeitsplatzsicherheit und ethische Anforderungen (OECD 2017b, p. 81). Zudem kommt es zu einer immer stärkeren Einschränkung beim Einsatz von Beamten in Kernfunktionen des Staates, wobei selbst in TopPositionen und im Bereich des mittleren Managements nicht mehr nur ausschließlich Beamte verwendet werden. In bestimmten Funktionen überwiegt mittlerweile sogar der Einsatz von Angestellten, so zum Beispiel bei administrativen und technischen Funktionen sowie beim Einsatz von IT- Experten (OECD 2017b, p. 81).

Für Deutschland lässt sich feststellen, dass der Beamtenstatus im System des deutschen öffentlichen Dienstes kaum in Gefahr geraten dürfte. Während in anderen Staaten pragmatische und ökonomische Argumente in der Diskussion eine zentrale Rolle spielen, wird die Debatte über die Zukunft des Beamtentums in Deutschland vor allem durch verfassungsrechtliche, rechtsphilosophische und historische Argumente dominiert, eine ernsthafte Abschaffung des Beamtenstatus wird kaum erwogen.

Insgesamt erscheint das klassische Beamtenmodell als ein Ansatz der klassischen Moderne, der in einer postmodernen Gesellschaft der Singularitäten (Reckwitz 2017) zunehmend seiner Substanz beraubt wird. Exemplarisch lässt sich dies beim Streikrecht für Beamte feststellen. Zwar wird in fast allen Mitgliedstaaten an einem Streikverbot für Beamte festgehalten, allerdings nur für bestimmte Funktionen, die hoheitliche Tätigkeiten ausüben. Das Streikrecht für Beamte wird (fast) europaweit als ein Grundrecht gesehen. Insgesamt wird die Substanz des klassischen Beamtentums europaweit langsam aufgezehrt, wie z. B. in den letzten Jahren auch in der Schweiz sowie partiell in Österreich. Diese Entwicklung setzt sich fort, weil es den Verteidigern des klassischen Beamtentums an empirisch abgesicherten Fakten mangelt, warum die klassischen Strukturprinzipien des Beamtentums beibehalten werden sollten.

Dennoch: Die öffentlichen Dienste haben nach wie vor überall das gleiche Ziel: der Demokratie, dem Recht und den Bürgern zu dienen. Damit besteht weiterhin eine kritische Positionierung der Verwaltung im Verhältnis zur politischen Macht sowie zu den Individualinteressen. In ihren vergleichenden Studien beklagen Mayer-Sahling et al. (2016) eine zunehmende Politisierung in den öffentlichen Diensten sowie Mungiu-Pippidi und Johnston (2017) eine weltweite Zunahme von Korruption. Diese Trends betreffen, mit unterschiedlicher Intensität, alle Systeme des öffentlichen Dienstes. 
Angesichts der ohnehin wachsenden Herausforderungen an (Rechts-) Staat und Demokratie bekommt somit die Feststellung von Raadschelders et al. (2015, p. 369), wonach das 21. Jahrhundert Staatsbedienstete mehr und dringender benötigt denn jemals zuvor, eine neue Bedeutung. Dennoch bleibt die Antwort auf die konkrete Ausgestaltung des nationalen öffentlichen Dienstes eng mit der nationalen Tradition verbunden. Trotz seiner zunehmenden Sonderstellung in Europa werden der deutsche Beamtenstatus und das deutsche Beamtenrecht nur sehr bedingt zu einer umfassenden Reform gedrängt. Allerdings empfiehlt sich ein Blick über die (deutschsprachigen) Grenzen, da Deutschland von internationalen Reformerfahrungen (sehr) profitieren kann.

\section{Literatur}

Auer, Astrid et al. 1996. Civil Services in the EU of 15, Maastricht: European Institute of Public Administration.

Bossaert, Danielle et al. 2001. Civil Services in the EU of 15: Trends and New Developments. Maastricht: European Institute of Public Administration.

Brunner, Dorothea. 2009. Die Wahrnehmung der Lohndisparität im Unternehmen und deren Wirkung auf die Kündigungsabsicht. München/Mering: Rainer Hampp.

Demmke, Christoph. 2016. Doing more with less? The Future of the Government Workforce. Frankfurt/M.: Peter Lang.

Demmke, Christoph, und Timo Moilanen. 2010. Civil Services in the EU of 27. Frankfurt/M.: Peter Lang.

Demmke, Christoph, und Timo Moilanen. 2013. Government Transformation and the Future of Public Employment. Frankfurt/M.: Peter Lang.

European Commission. 2018. A comparative overview of public administration characteristics and performance in EU 28. Brussels: European Commission.

European Foundation for the Improvement of Living and Working Conditions. 2012. Work Organisation and Innovation. Dublin: Eurofound.

Kuhlmann, Sabine, und Hellmut Wollmann. 2014. Introduction to Comparative Public Administration. Cheltenham: Edward Elgar.

Matthews, Felicity. 2012. Governance and State Capacity. In The Oxford Handbook of Governance, Eds. David Levi Faur. Oxford: Oxford University Press DOI:10.1093/oxfordhb/9780199560530.013.0020. 
Meyer-Sahling, Jan-Hinrik, Will Lowe, and Christian van Stolk. 2016. Silent professionalization: EU integration and the professional socialization of public officials in Central and Eastern Europe. European Union Politics 17(1): 162-183.

Meyer-Sahling Jan-Hinrik, and Kim Sass Mikkelsen. 2016. Civil Service Laws, Merit, Politicization, and Corruption: The Perspective of public Officials from Five East European Countries. Public Administration 94(4): 1105-1123. http://dx.doi.org/10.1111/padm.12276.

Mungiu-Pippidi, Alina, and Michael Johnston. 2017. Transitions to Governance, Cheltenham: Edward Elgar.

Guest, David. 2014. Employee engagement: a skeptical analysis. In Journal of Organisational Effectiveness, People and Performance 41 (2): 291-314.

OECD. 2010. Innovative Workplaces. Paris: OECD.

OECD. 2016. Engaging Public Employees for a High Performing Civil Services, Paris: OECD.

OECD. 2017a. Governance at a Glance, Paris: OECD.

OECD. 2017b. Skills for a High-Performing Civil Service, Paris: OECD.

Purcell, John. 2014. Disengaging from engagement. In Human Resource Management Journal 24 (3): 241-254.

Reckwitz, Andreas. 2017. Die Gesellschaft der Singularitäten, Berlin: Suhrkamp

van der Meer, Frits, Jos Raadschelders, and Theo Toonen. 2011. Civil Service Systems in Western Europe. Cheltenham/Aldershot: Edward Elgar.

Raadschelders, Jos, Theo Toonen, and Frits van der Meer. 2015. Civil Servants in the Enabling Framework State of the $21^{\text {st }}$ century. In: Comparative Civil Service Systems in the 21st Century. Hrsg. Raadschelders, Jos, Theo Toonen, and Frits van der Meer, 354-369.Basingstoke: Palgrave MacMillan.

van der Meer, Frits, Jos Raadschelders und Theo Toonen. 2015. Comparative Civil Service Systems in the 21st Century, Basingstoke: Palgrave MacMillan.

Verheijen, Tony et al. 1999. Civil Service Systems in Central and Eastern Europe. Cheltenham: Edward Elgar. 